\title{
Error Monitoring Using External Feedback: Specific Roles of the Habenular Complex, the Reward System, and the Cingulate Motor Area Revealed by Functional Magnetic Resonance Imaging
}

\author{
Markus Ullsperger and D. Yves von Cramon \\ Max Planck Institute of Cognitive Neuroscience, D-04103 Leipzig, Germany
}

\begin{abstract}
The dopaminergic system has been shown to be involved in the processing of rewarding stimuli, specifically of errors in reward prediction, in animal studies as well as in recent neuroimaging studies in humans. Furthermore, a specific role of dopamine in the human homolog of the rostral cingulate motor area (rCMA) was proposed in a recent model of error detection. Negative feedback as well as self-detected errors elicit a negative event-related brain potential probably generated in the rCMA. We performed two experiments using functional magnetic resonance imaging to investigate the brain activity related to negative and positive feedback in a dynamically adaptive motion prediction task. Whereas positive feedback raised hemodynamic activity in the ventral striatum (nucleus accumbens), negative feedback activated the rCMA, the inferior anterior insula, and the epithalamus (habenular complex). These data demonstrate the role of the habenular complex in the control of the human reward system, a function previously hypothesized on the basis of animal research. The rCMA reacted only to errors with negative feedback but not to errors without feedback, which ruled out an influence of response conflict or uncertainty on its role in error detection by external signals.
\end{abstract}

Key words: error detection; performance monitoring; reward; feedback; CMA; habenula; fMRI

\section{Introduction}

Goal-directed behavior and skill acquisition require continuous performance monitoring. Good performance is reinforced; deviations from the goals (errors) call for remedial actions and strategy adjustments. Although action slips resulting from premature responses can be internally detected by the individual, mistakes attributable to insufficient knowledge are recognized by their consequences (external feedback) (Rabbitt, 1966; Reason, 1990). It has been shown that even abstract positive feedback activates the same brain structures as primary reward, in particular the ventral striatum with the nucleus accumbens (Elliott et al., 2000). Several lines of evidence suggest an important role of the dopaminergic system in reward processing, more specifically in signaling errors in reward prediction (Schultz, 2000, 2002; Schultz and Dickinson, 2000; Pagnoni et al., 2002). Unpredicted primary or conditioned secondary reward stimuli elicit a strong phasic dopaminergic response. In contrast, after omission of expected rewarding stimuli, the basal dopaminergic activity in the ventral tegmental area (VTA) and substantia nigra (SN) temporarily ceases.

Electrophysiological and hemodynamic studies suggest a specific role of the rostral cingulate motor area (rCMA) in error detection and generation of an error-specific event-related brain

\footnotetext{
Received Dec. 19, 2002; revised March 5, 2003; accepted March 7, 2003.

This work was supported with help from F. Szymanowski, M. Naumann, A. Mempel, and C. Buschendorf in data collection, the contributions of J. Lepsien and A. Szameitat in task development, and the comments of two anonymous reviewers on a previous version of this manuscript.

Correspondence should be addressed to Markus Ullsperger, Max Planck Institute of Cognitive Neuroscience, Stephanstrasse 1a, D-04103 Leipzig, Germany. E-mail: ullsperg@cns.mpg.de.

Copyright $\odot 2003$ Society for Neuroscience $\quad$ 0270-6474/03/234308-07\$15.00/0
}

potential (ERP), the error-related negativity (ERN) (Falkenstein et al., 1990; Gehring et al., 1993; Carter et al., 1998; Ullsperger and von Cramon, 2001). An ERP component of identical scalp topography was described for external negative feedback on errors undetectable for participants for lack of sufficient information (Miltner et al., 1997). According to a recent model, the ERN results from disinhibited neuronal activity in the rCMA attributable to phasic depression of the dopaminergic activity on errors (Holroyd and Coles, 2002). Self-detection of error, as well as external negative feedback in hard-to-detect errors, predicts the nonoccurrence of reward, which should result in decreased dopamine release.

It is still rather unclear how the mesencephalic dopaminergic neurons are inhibited when expected rewards do not occur. Animal studies provide evidence that the VTA and SN receive inhibiting neurons from a structure of the dorsal medial thalamus (epithalamus) called the habenula, because of its morphological resemblance to a rein. Electrical stimulation of the habenular nuclei causes inhibition of $\sim 85-90 \%$ of the dopamine neurons in the VTA and SN in rats (Christoph et al., 1986). In contrast, habenular lesions result in increased dopamine turnover in the nucleus accumbens, striatum, and prefrontal cortex, reflecting an activation of the dopaminergic system (Lisoprawski et al., 1980; Nishikawa et al., 1986). The habenular complex receives fibers from the basal forebrain, medial striatum, and anterior hypothalamus via the stria medullaris thalami. The main efferent pathway is the fasciculus retroflexus of Meynert projecting to the interpeduncular nucleus, VTA and SN, medial raphe complex, locus ceruleus, and central gray (Scheibel, 1997). These anatomical findings suggest high importance of the habenular complex as a 


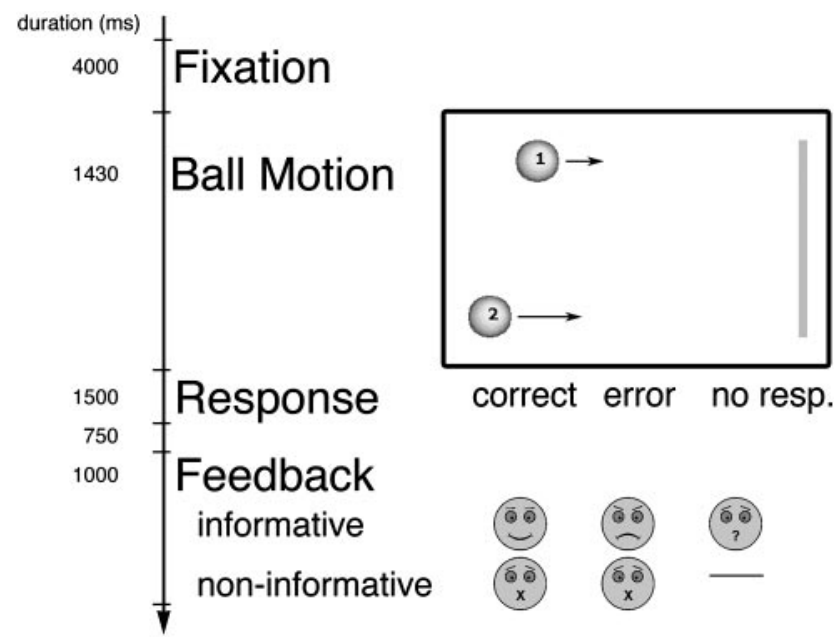

Figure 1. Timing of the dynamically adaptive motion prediction task ("first-over-the-finishline-task"). Note that the noninformative stimuli instead of feedback were presented only in experiment 2 .

critical modulatory relay between the limbic forebrain structures and the midbrain.

Our study aimed at investigating error processing on the basis of external feedback using functional magnetic resonance imaging (fMRI). We hypothesized a larger hemodynamic activity of the epithalamus (habenular complex) on errors with negative feedback and a lower hemodynamic activity on correct responses with positive feedback. Moreover, based on electrophysiological findings (Miltner et al., 1997), we predicted selectively increased rCMA activity for negative feedback on mistakes.

\section{Materials and Methods}

Participants and task. Sixteen healthy right-handed volunteers participated in each experiment (experiment 1: nine females, 21-28 years of age, mean age, 24.1; experiment 2: eight females, 20-33 years of age, mean age, 25). Informed consent was obtained from each participant according to the declaration of Helsinki. The experiments were approved by the University of Leipzig Ethics Committee. Stimuli were presented using Presentation 0.45 (Neurobehavioral Systems, San Francisco, CA) and appeared on a back-projection screen mounted inside the scanner bore, which was viewed through mirror glasses. A new dynamically adaptive motion prediction (DAMP) task was applied in both experiments (Fig. 1). During each trial, participants observed a short sequence of the motion of two balls that moved from different respective starting points (in one-half of the screen) and different speeds toward a finish line on the other side. After $1.43 \mathrm{sec}$, the balls disappeared (still far from the finish line), and the question "which ball?" was presented on the screen. The task was to predict which ball would first cross the finish line and to indicate the decision by a button press. During the experiments, task difficulty (operationalized as the time difference of arrival of the two balls at the finish line) was dynamically adapted to each participant's behavior, such that the error rate was constantly kept at $\sim 37 \%$. Therefore, participants were highly uncertain about whether their prediction was correct. A feedback about correctness of the prediction (a smiley face) was presented $750 \mathrm{msec}$ after the response. The next trial started after a fixation period of at least $4000 \mathrm{msec}$. To keep the error rate high during the first trials of the experiments, individual difficulty levels were determined in a training session (100 trials and only informative feedback) that was performed during the anatomical scans.

In experiment 1 , we investigated the hemodynamic response elicited by feedback stimuli that were informative on all trials (i.e., errors were always followed by negative feedback, and correct responses were followed by positive feedback). In experiment 2 , we introduced an additional noninformative stimulus that occurred instead of the informative feedback with a probability of $26.5 \%$ on correct and incorrect responses each. It contained no information on whether the response was correct (i.e., the smiley face had an " $x$ " instead of a mouth) (Fig. 1). Experiment 1 consisted of 120 trials and 12 randomly interspersed nonevents; experiment 2 consisted of 200 trials and 20 nonevents.

Image acquisition and analysis. Imaging was performed at $3 \mathrm{~T}$ on a Bruker (Ettlingen, Germany) Medspec 30/100 system equipped with the standard bird cage head coil. Sixteen functional slices were obtained parallel to the anterior commissure-posterior commissure (AC-PC) line (thickness, $5 \mathrm{~mm}$; spacing, $1 \mathrm{~mm}$ ) using a single-shot gradient echoplanar imaging (EPI) sequence (repetition time, 2 sec; echo time, 30 msec; $64 \times 64$ pixel matrix; flip angle, $90^{\circ}$; field of view, $192 \mathrm{~mm}$ ) sensitive to blood-oxygen level-dependent contrast. Trials occurred at multiple, systematically offset time points (range, $0-0.5 \mathrm{sec}$ ) in relation to the image acquisition to improve temporal resolution (Josephs et al., 1997; Miezin et al., 2000). Before the functional runs, anatomical modified driven equilibrium Fourier transform (MDEFT) and EPI-T1 slices in the plane with functional images were collected. Susceptibility artifacts (image distortion and signal loss) were only present in the orbitofrontal and frontopolar regions, which, therefore, are not discussed in this paper (see Wansapura et al., 1999, for more details regarding susceptibility artifacts at $3 \mathrm{~T}$ ).

Data processing was performed using the software package Leipzig Image Processing and Statistical Inference Algorithms (Lohmann et al., 2001). Functional data were corrected for motion artifacts and slicetime acquisition differences using sinc-interpolation. Signal changes and baseline-drifts were removed by applying a temporal high-pass filter with a cutoff frequency of $1 / 200 \mathrm{~Hz}$. Spatial smoothing was applied using a Gaussian filter with $5.65 \mathrm{~mm}$ full width at half maximum (FWHM).

To align the functional data slices with a three-dimensional stereotactic coordinate reference system, a rigid linear registration with $6 \mathrm{df}$ (three rotational and three translational) was performed. The rotational and translational parameters were acquired on the basis of the MDEFT and EPI-T1 slices to achieve an optimal match between these slices and the individual three-dimensional reference data set that was acquired for each subject during a previous scanning session. The MDEFT volume data set with 160 slices and $1 \mathrm{~mm}$ slice thickness was standardized to the Talairach stereotactic space (Talairach and Tournoux, 1988). The rotational and translational parameters were subsequently transformed by linear scaling to a standard size. The resulting parameters were then used to transform the functional slices using trilinear interpolation so that the resulting functional slices were aligned with the stereotactic coordinate system, generating output data with a spatial resolution of $3 \mathrm{~mm}^{3}$. The statistical analysis was based on a least squares estimation using the general linear model for serially autocorrelated observations (Friston et al., 1995; Worsley and Friston, 1995; Aguirre et al., 1997; Zarahn et al., 1997). The design matrix was generated with a synthetic hemodynamic response function (Friston et al., 1998). The model equation, including the observation data, the design matrix, and the error term, were convolved with a Gaussian kernel of dispersion of $4 \mathrm{sec}$ FWHM. The effective degrees of freedom were estimated as described by Worsley and Friston (1995). Contrasts between negative and positive feedback conditions were calculated. The resulting contrast images of all participants were subjected to a voxel-wise one-sample $t$ test that indicated whether observed differences between conditions were significantly distinct from zero (Holmes and Friston, 1998). Resulting $z$-maps were thresholded at $z>3.09$, uncorrected. Event-related analysis was performed on the onset of the feedback stimuli. In addition, averaged time courses of the hemodynamic response for all conditions were investigated in experiment 2. The mean amplitudes of the hemodynamic response (percentage signal change related to the mean signal of the entire signal) were submitted to repeated-measures ANOVAs in which all effects with $>1 \mathrm{df}$ in the numerator were adjusted according to the formula put forth by Greenhouse and Geisser (1959).

Neuroanatomical criteria. To ensure highest anatomical precision, we independently determined the coordinates of the habenular complex in the individual anatomical data sets (inter-rater reliability, 98.4\%). For habenular nuclei, the time courses at those individual coordinates were 
computed, and for all other regions under investigation, the coordinates of the maximal $z$-values in the group statistics were used.

We defined anatomical regions at the frontomedian wall according to the literature on homolog medial premotor areas in human and nonhuman primates (Vogt et al., 1995; Picard and Strick, 1996; Vorobiev et al., 1998). The Talairach coordinates of the regions are depicted in Picard and Strick's (1996) review and served as a reference for localization of the activation in our study. Activation was defined as falling into the presupplementary motor area (pre-SMA) when they were located anterior to the coronal plane through the anterior commissure $(y>0)$ (Vorobiev et al., 1998), and $>45 \mathrm{~mm}$ above the AC-PC plane $(z>45)$. The anterior border of the pre-SMA is less well defined in the literature. Traditionally, it is identified as the border between Brodmann area (BA) 6 and BA 8 (Picard and Strick, 1996). The rCMA or rostral cingulate zone is primarily buried in the cingulate sulcus and located anterior to the coronal plane through the anterior commissure and posterior to the genu of the corpus callosum. It comprises BA $24 \mathrm{c}^{\prime}$ and might extend into BA $32^{\prime}$, as indicated by Picard and Strick (1996).

\section{Results}

\section{Behavioral data}

Reaction times were significantly shorter for correct predictions than for erroneous predictions in both experiments (experiment $1: M=633.3$ and $661.2 \mathrm{msec}, F_{(1,15)}=16.03, p<0.005$; experiment $2: M=524.5$ and $\left.557.5 \mathrm{msec}, F_{(1,15)}=18.81, p<0.001\right)$. Because of dynamic difficulty adjustments, in both experiments, error rates amounted to $36.8 \%$ (SEM in experiments 1 and 2, 1.4 and 1.0 , respectively).

In the debriefing of experiment 2, participants rated the overall certainty of their responses during the main experiment and training block on a scale ranging from 0 to 4 . During training, difficulty was lower and participants' responses were erroneous only $22.8 \%$ of the time (SEM, 0.8 ). In concordance with the higher error rates during the main experiment, the certainty at response was significantly reduced $(p<0.001)$ compared with the training, suggesting that participants' performance monitoring depended on feedback evaluation.

\section{Experiment 1: fMRI data}

We investigated the hemodynamic response elicited by feedback stimuli that were informative on all trials (i.e., errors were always followed by negative feedback and hits by positive feedback). Although on negative feedback, hemodynamic activity was higher in the human homolog of the rCMA, pre-SMA, anterior inferior insula, and epithalamus (habenular complex), for positive feedback, the ventral striatum (nucleus accumbens) and the putamen were more activated (see Table 1 for list of activations). Before functional data analysis, the habenular complex was independently identified by the two authors in each subject's anatomical MRI. The habenular negative-feedback-related activation was clearly located within the area resulting from overlapping the single subject coordinates of the habenular nuclei (Fig. 2).

Activity related to negative feedback on errors in experiment 1 could reflect the response of the brain to the omission of reward. However, the reaction times on error trials were significantly longer than those on correct trials, suggesting a higher uncertainty or response conflict during errors. Thus, the activation on negative feedback could also be a correlate of response conflict and uncertainty about which response would be rewarding. Temporal overlap of the hemodynamic response on reaction and feedback does not allow disentanglement of activity related to negative feedback from conflict- and uncertainty-related activations. One way to firmly establish that the feedback-related effects are not attributable to differences in uncertainty or processing
Table 1. List of activations revealed by contrasting errors with negative feedback versus correct trials with positive feedback in experiment 1

\begin{tabular}{llrrrr}
\hline & & \multicolumn{3}{l}{ Talairach coordinates } & \\
\cline { 3 - 4 } Side & Brain region & $x$ & $y$ & $z$ & Zscore \\
\hline Negative $>$ positive feedback & & & & \\
$\mathrm{R}$ & rCMA (BA 24c') & 6 & 35 & 3.81 \\
$\mathrm{R}$ & Pre-SMA (BA 6) & 0 & 13 & 53 & 3.89 \\
$\mathrm{~L}$ & Anterior inferior insula (BA 13/14) & -37 & 10 & -3 & 4.34 \\
$\mathrm{R}$ & Anterior inferior insula (BA 13/14) & 42 & 7 & 0 & 4.28 \\
$\mathrm{~L}$ & Superior bank of IFS (BA 9) & 48 & 19 & 32 & 3.79 \\
$\mathrm{~L}$ & Inferior precentral sulcus (BA 6) & 54 & 10 & 26 & 3.48 \\
$\mathrm{R}$ & Anterior SFS (BA 8) & 15 & 40 & 29 & 3.90 \\
$\mathrm{~L}$ & Anterior IPS (BA 7/40) & -35 & -46 & 41 & 3.90 \\
$\mathrm{R}$ & IPL (BA 40) & 51 & -49 & 32 & 4.75 \\
$\mathrm{~L} / \mathrm{R}$ & Habenular complex (bilateral) & $3 /-5$ & -25 & 8 & 4.05 \\
$\mathrm{~L}$ & Thalamus (ventrolateral/anterior Nc) & -11 & -10 & 11 & 4.44 \\
$\mathrm{R}$ & Thalamus (ventrolateral/anterior Nc) & 12 & -10 & 14 & 4.08 \\
$\mathrm{R}$ & Thalamus (ventrolateral Nc) & 9 & -10 & 5 & 3.86 \\
$\mathrm{~L}$ & Thalamus (laterodorsal Nc) & -14 & -22 & 21 & 3.89 \\
Positive $>$ negative feedback & & & & \\
$\mathrm{L}$ & Ventral striatum (Nc accumbens) & -17 & 7 & -5 & 4.00 \\
$\mathrm{~L}$ & Putamen & -22 & -1 & 14 & 3.80 \\
$\mathrm{R}$ & Putamen & 24 & -19 & 8 & 4.00 \\
\hline
\end{tabular}

IFS, Inferior frontal sulcus; IPL, inferior parietal lobule; IPS, intraparietal sulcus; SFS, superior frontal sulcus; $\mathrm{Nc}$ nucleus; L, left; R, right. Asterisk indicates pre-SMA not significantly activated with reaction time as regressor.

time of the task was to reanalyze the data with the reaction time as a regressor. We found the same activation pattern as reported in Table 1, except for the finding that the pre-SMA did not show significant activity in this reanalysis.

\section{Experiment 2: fMRI data}

Another way to disentangle feedback-related activity from uncertainty-related activity was chosen in experiment 2 , in which a noninformative stimulus occurred with a probability of $26.5 \%$ on each correct and incorrect response instead of the informative feedback. Activation related to response conflict or uncertainty should occur independently, regardless of whether feedback or noninformative stimuli were presented (i.e., it should be highest on errors regardless of whether it was followed by a negative feedback). In contrast, brain activity related to negative feedback processing should be highest exclusively on errors followed by informative feedback.

In contrasting errors with informative (negative) feedback versus correct trials with informative (positive) feedback, the results from experiment 1 were replicated (Table 2). In Figure 3, the mean signal changes of the hemodynamic responses for all four conditions (correct and error times presence and absence of feedback) are depicted for several regions of interest (ROIs). Additional analyses revealed that in these ROIs, except for the preSMA, there was an interaction between the response type (Resp, two levels) and feedback occurrence (Feedb, two levels) (Table 3) of the factors.

In the ventral striatum (nucleus accumbens) (Fig. $3 d$ ), a reliable signal increase was found with positive feedback only on correct trials (i.e., the signal change was significantly larger than zero; $\left.T_{(15)}=6.38 ; p<0.0001\right)$.

Mean amplitude data from the rCMA and insula (Fig. 3a,e) were subjected to repeated-measure ANOVAs with the factors Resp and Feedb that revealed main effects of both factors (rCMA Resp, $F_{(1,15)}=4.87, p<0.05$; rCMA Feedb, $F_{(1,15)}=7.42$, $p<0.05$; insula Resp, $F_{(1,15)}=27.51, p<0.0001$; insula Feedb, $F_{(1,15)}=4.09, p<0.062$ ) and a Resp by Feedb interaction (rCMA, $F_{(1,15)}=4.62, p<0.05$; insula, $\left.F_{(1,15)}=8.61, p<0.05\right)$. Planned 
a)
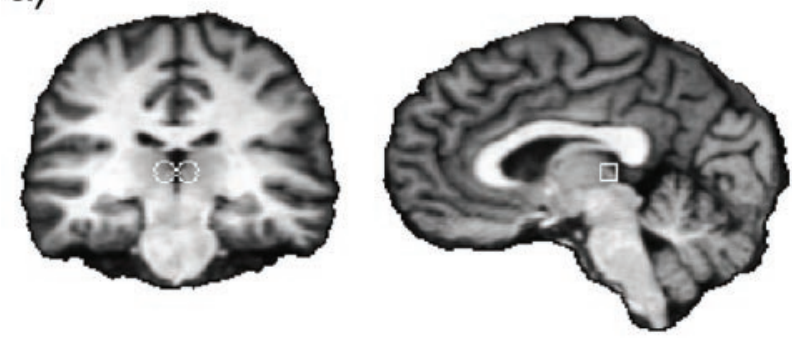

b)
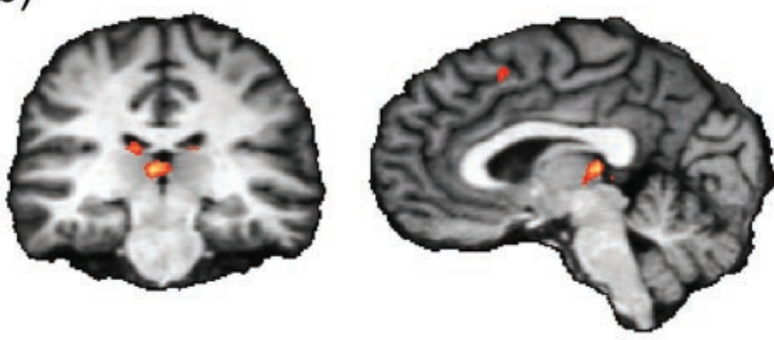

3.1 4.7

Figure 2. Activation of the epithalamus in experiment 1. $a, 0$ verlap area resulting from variability of the habenular complex across subjects. $b, z$-map of activation. From left to right: coronal, sagittal, and horizontal slices at $x=-2, y=-25$, and $z=8$.

Table 2. List of activations revealed by contrasting errors with negative feedback versus correct trials with positive feedback in experiment 2

\begin{tabular}{|c|c|c|c|c|c|}
\hline \multirow[b]{2}{*}{ Side } & \multirow[b]{2}{*}{ Brain region } & \multicolumn{3}{|c|}{ Talairach coordinates } & \multirow[b]{2}{*}{ Zscore } \\
\hline & & $x$ & $y$ & $z$ & \\
\hline \multicolumn{6}{|c|}{ Negative $>$ positive feedback } \\
\hline $\mathrm{R}$ & rCMA (BA 24c') & 4 & 18 & 35 & 3.69 \\
\hline $\mathrm{R}$ & Pre-SMA (BA 6) & 4 & 15 & 53 & 3.79 \\
\hline$L$ & Anterior inferior insula (BA 13/14) & -37 & 15 & -3 & 4.20 \\
\hline $\mathrm{R}$ & Superior bank of IFS (BA 9) & 39 & 10 & 29 & 3.75 \\
\hline$L / R$ & Habenular complex & $-5 / 6$ & -25 & 8 & 3.66 \\
\hline $\mathrm{R}$ & Thalamus (ventrolateral Nc) & 15 & -13 & 14 & 4.10 \\
\hline $\mathrm{L}$ & Thalamus (posterolateral Nc) & -12 & -16 & 14 & 3.71 \\
\hline $\mathrm{R}$ & Cuneus (BA 18) & -2 & -73 & 20 & 3.65 \\
\hline \multicolumn{6}{|c|}{ Positive $>$ negative feedback } \\
\hline \multirow[t]{2}{*}{ L } & Ventral striatum (Nc accumbens) & -13 & 6 & -3 & 4.38 \\
\hline & Ventral striatum (ventral caudate) & -7 & 15 & 0 & 4.13 \\
\hline $\mathrm{R}$ & Ventral striatum (Nc accumbens) & 8 & 8 & -3 & 3.87 \\
\hline $\mathrm{L}$ & IPS (horizontal branch, BA 7/40) & -29 & -52 & 41 & 3.76 \\
\hline$L$ & Caudate Nc & -14 & -13 & 23 & 4.32 \\
\hline
\end{tabular}

IFS, inferior frontal sulcus; IPS, intraparietal sulcus; Nc, nucleus; L, left; R, right.

comparisons confirmed that in both ROIs, errors with informative feedback evoked significantly higher hemodynamic activity than all other conditions. Although the signal for errors with negative feedback was larger than that for errors without feedback (rCMA, $F_{(1,15)}=8.56, p<0.05$; insula, $F_{(1,15)}=8.76, p<$ $0.01)$ and correct trials with feedback ( $\mathrm{rCMA}, F_{(1,15)}=12.41, p<$ 0.005 ; insula, $\left.F_{(1,15)}=29.07, p<0.0001\right)$, no significant signal difference was found between correct trials with and without feedback and errors without feedback $(p>0.38)$.

The data from the pre-SMA (Fig. $3 b$ ) were subjected to the same ANOVA, which gave rise only to a main effect of Resp $\left(F_{(1,15)}=12.25 ; p<0.005\right)$, reflecting errors leading to higher activation of the pre-SMA than correct responses regardless of the presence or absence of the feedback.

Finally, the same analysis performed on the data from the

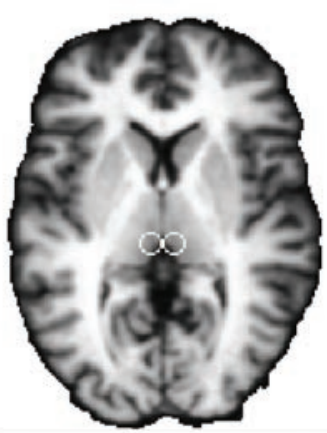

habenular complex (Fig. 3c) gave rise to a Resp by Feedb interaction $\left(F_{(1,15)}=7.29\right.$; $p<0.05)$. Here, single-condition comparisons showed that errors with negative feedback led to significantly higher hemodynamic responses than errors without feedback $\left(F_{(1,15)}=6.22 ; p<0.05\right)$ and correct responses with positive feedback $\left(F_{(1,15)}=6.41 ; p<0.05\right)$. Furthermore, correct trials without feedback showed a tendency to activate the habenular complex more than correct trials with positive feedback $\left(F_{(1,15)}=4.00 ; p<0.064\right)$ and errors without feedback $\left(F_{(1,15)}=3.15\right.$; $p<0.097)$.

\section{Discussion}

The present two studies aimed at investigating performance monitoring under conditions when the individual cannot detect the errors because of lack of knowledge. The resemblance of the negative ERP on feedback with the ERN (Miltner et al., 1997; Luu et al., 2003) suggests that similar networks are involved in error detection based on external feedback as in self-detection of action slips. We used a DAMP task, making sure that the difficulty was tailored to each individual such that high uncertainty about whether the response was correct was induced. Hence, for evaluation of the responses and strategy adjustment, participants were dependent on the feedback. Because error trials might also involve a higher degree of uncertainty and response conflict preceding the feedback, and that response conflict may account for similar activation differences (cf. Carter et al., 1998), we performed experiment 2 , in which on a proportion of trials, no feedback was given. Those trials would involve uncertainty and response conflict but not the negative-feedback-associated activity.

\section{Interactions with the reward processing system}

In summary, the results from the two studies provide several new insights into the mechanisms involved in performance monitoring and reward processing and point at the interfaces between these cognitive functions. The ventral striatum was activated only when positive feedback occurred. This supports the fact that the ventral striatum is engaged when rewards or positive feedback occur (Elliott et al., 2000; Berns et al., 2001; Pagnoni et al., 2002; Volz et al., 2003), probably because of phasic dopamine release (Schultz, 2000, 2002; Schultz and Dickinson, 2000). It is also compatible with the view that the attribution "incentive salience" to the stimuli (i.e., that the stimuli are "wanted") (cf. Berridge and Robinson, 1998) is reflected by dopamine release in the nucleus accumbens. Our results seem to be inconsistent with previous findings (Horvitz et al., 1997; Horvitz, 2000) that nonrewarding salient events may result in dopamine release in the nucleus accumbens, suggesting that less frequent noninformative and negative feedback stimuli might induce higher activity in the ventral striatum, which was not found in our data. An explanation could be that the stimuli in this research are not comparable with nonconditioned stimuli, as used by Horvitz et al. (1997), because their association to reward, nonoccurrence of reward, and uncertainty about reward, respectively, was established by instruction and experienced throughout the experiment. 
Furthermore, these data highlight processes in other structures that come into play when expected reward fails to occur (e.g., during negative feedback). They clarify the role of the habenular complex that has primarily been neglected in research in humans (partly because of the fact that it is almost never selectively damaged). When an error was made and negative feedback was received, the habenular nuclei seemed to be activated most. Similar activity was observed when correct responses were not followed by informative (positive) feedback (i.e., there was an interaction of response type and the occurrence of informative feedback). To understand this interaction, one needs to keep in mind that the habenula inhibits the midbrain nuclei. Many neuromodulator systems are influenced by fibers from the habenular complex (Scheibel, 1997). We focus the discussion on the dopamine system, which seems to be the most involved in reward prediction and error processing (Schultz, 2000, 2002; Holroyd and Coles, 2002). It is conceivable that the higher engagement of the habenula observed in the experiment reduces the probability of phasic dopamine release in the reward system. This inhibitory function seems to be based on an integration of reward expectancy and the actual occurrence of reward or punishment. In this experiment, the actual reward relevant for goal-directed behavior is the knowledge that the response was correct, symbolized here by the smiley face. The reward expectancy is not only dependent on the global frequency of positive rewards in the experiment but also on the certainty about the correctness of the current response. The longer reaction times suggested that uncertainty was higher during error trials than correct responses, leading to lower reward expectancy. For trials without feedback information (i.e., without reward or punishment), this could have resulted in lower habenular activity for errors than correct trials (Fig. $3 c$ ), such that the VTA and SN are less inhibited (if it occurred, a feedback resulting in a phasic dopamine signal would be highly informative during the uncertain error trials). However, negative feedback on errors assures the participant that no reward (in the form of knowing that the response was correct) can be received in the current trial. Because before the feedback reward prediction was not zero, this reflects a negative error in reward prediction. The accompanying increased habenular activity might indicate an increased inhibition of the dopaminergic midbrain nuclei resulting in decreased dopamine output, as reported for nonoccurring rewards (Schultz and Dickinson, 2000; Schultz, 2002). According to the model proposed by Holroyd and Coles (2002), this decreased dopamine release can result in higher activity in the rCMA, as was the case in this experiment during errors with negative feedback. In contrast, for correct trials, reward expectancy was slightly higher than during errors because of higher certainty. However, the global frequency of positive feedback in experiment 2 was only $45.2 \%$; thus, reward expectancy was not very high, even during correct trials. Therefore, positive feedback would still be informative for the system, but less so than during the error trials involving higher uncertainty (this might explain the relatively high habe- b)
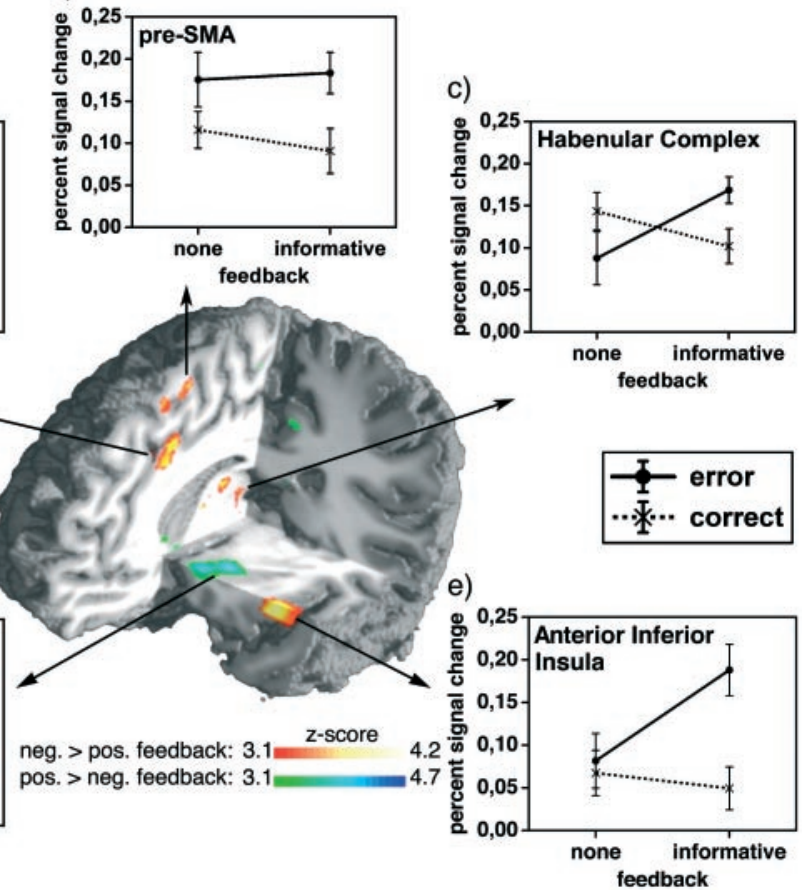

g. > pos. feedback: 3.1 z-score pos. $>$ neg. feedback: 3.1

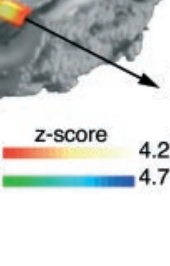

Figure 3. Activations in experiment 2. The central picture shows the z-map resulting from contrasting negative and positive informative feedback. $a-d$, Signal change of the hemodynamic response for correct and error trials with and without informative

Table 3. Regions of interest showing interactions between feedback presence and response type

\begin{tabular}{llrrr}
\hline & & \multicolumn{3}{c}{ Talairach coordinates } \\
\cline { 3 - 5 } Side & Brain region & $x$ & $y$ & $z$ \\
\hline Interaction response type $\times$ presence of feedback & & 18 & 35 \\
$\mathrm{R}$ & $\mathrm{rCMA}$ & -37 & 15 & -3 \\
$\mathrm{~L}$ & Anterior inferior insula & -13 & 6 & -3 \\
$\mathrm{~L}$ & Ventral striatum (Nc accumbens) & 8 & 8 & -3 \\
$\mathrm{R}$ & Ventral striatum (Nc accumbens) & $-5 / 6$ & -25 & 8 \\
$\mathrm{~L} / \mathrm{R}$ & Habenular complex & & &
\end{tabular}

Nc, Nucleus; L, left; R, right.

nular activity during correct trials without feedback). Therefore, positive feedback on correct trials revealed a positive error in reward prediction. This error in reward prediction is reflected in the decrease in the habenular activity when correct trials were followed by positive feedback, thus disinhibiting the dopaminergic midbrain areas. Bearing in mind the anatomical connections of the habenular complex, this interpretation is consistent with the view that the VTA and SN react with phasic changes of dopamine release to errors in reward prediction (i.e., with dopamine release on unexpected rewards and a decrease in activity on nonoccurring predicted rewards) (Schultz and Dickinson, 2000; Pagnoni et al., 2002). It seems that the difference in the habenular activity between informative and noninformative activity correlates with the error in reward prediction. However, fMRI is not able

to characterize the exact time course of the habenular activity, and in particular, it cannot disentangle phasic and tonic activity. Therefore, electrophysiological studies in primates might be of significant help in understanding the function of the epithalamus. 


\section{Areas on the frontomedian wall}

In experiment 2, the rCMA seems to specifically react on errors followed by informative (i.e., negative) feedback. It is important to note that the same area has been shown repeatedly to be involved in self-detection of errors (Carter et al., 1998; Ullsperger et al., 2001). It is conceivable that the rCMA is the generator not only of the ERN but also of the feedback-related negativity (Miltner et al., 1997; Luu et al., 2003). Thus, error detection based on the comparison of representations of the intended response and the actual response appears to involve mechanisms very similar to those seen for error detection based on external feedback. In both cases, error detection can lead to remedial actions and skill acquisition (Rabbitt, 1966; Reason, 1990). The role of the rCMA in this function is supported by the findings of Shima and Tanji (1998), demonstrating that CMA neurons respond only when reduced reward leads to a change in behavior (i.e., to remedial actions). A similar finding was reported recently for an fMRI study in humans (Bush et al., 2002). In this context, it would be interesting to investigate whether feedback induced performance gains suggestive of visuomotor learning, and whether this was related to changes in feedback-related brain activity. However, over the given time frame, there was no evidence for performance gains that should be reflected in increasing difficulty or shorter reaction times. Longer experiments with more trials will be needed to investigate this issue.

In contrast to the rCMA, the pre-SMA was activated by errors in general and even without negative feedback (i.e., without the individual's knowledge of a mistake). As mentioned above, erroneous trials seemed to involve higher uncertainty about what response to choose (higher response conflict and lower reward expectancy) than correct trials. This uncertainty persisted even after the response. The activation pattern supports the view that the pre-SMA is preferentially engaged by response conflict and/or uncertainty. The findings from experiment 2 are corroborated by the reanalysis of experiment 1 with reaction time as a regressor, which revealed that the pre-SMA activity correlated with the reaction time and thus with uncertainty. Furthermore, these findings are in accordance with recent studies investigating underdetermined responding (Elliott and Dolan, 1998; Volz et al., 2003). A similar functional dissociation of the rCMA (most activated during errors) and pre-SMA (most engaged by uncertainty and response conflict), as in our data, was shown in studies investigating self-detected errors (Ullsperger and von Cramon, 2001; Garavan et al., 2002).

Similarly, as with the rCMA, the anterior inferior insula was most activated by negative feedback. It could be speculated that this activity is correlated with accompanying autonomic responses to the negative emotional action of the feedback.

\section{Conclusion}

Our pair of experiments illustrates the close relationship between performance monitoring and reward processing. It shows that reward and nonoccurrence of reward activate different players in the network (the ventral striatum and the rCMA, respectively). The importance of the habenular complex in reward processing and influencing the dopaminergic system was demonstrated for the first time in humans. It appears that the habenula restrains the midbrain nuclei and plays a role in determining the error in reward prediction. As described previously by Scheibel (1997), the functional integrity of the epithalamus can be assumed to be relevant for psychiatric disturbances and drug abuse. In our opinion, the findings of this study suggest that measurements of single-unit activity in the habenula of primates would reveal in- teresting results on the tonic and phasic neuronal activity influencing the midbrain nuclei.

\section{References}

Aguirre GK, Zarahn E, D’Esposito M (1997) Empirical analysis of BOLD fMRI statistics, Vol II: spatially smoothed data collected under nullhypothesis and experimental conditions. NeuroImage 5:199-212.

Berns GS, McClure SM, Pagnoni G, Montague PR (2001) Predictability modulates human brain response to reward. J Neurosci 21:2793-2798.

Berridge KC, Robinson TE (1998) What is the role of dopamine in reward: hedonic impact, reward learning, or incentive salience? Brain Res Brain Res Rev 28:309-369.

Bush G, Vogt BA, Holmes J, Dale AM, Greve D, Jenike MA, Rosen BR (2002) Dorsal anterior cingulate cortex: a role in reward-based decision making. Proc Natl Acad Sci USA 99:523-528.

Carter CS, Braver TS, Barch DM, Botvinick MM, Noll D, Cohen JD (1998) Anterior cingulate cortex, error detection, and the online monitoring of performance. Science 280:747-749.

Christoph GR, Leonzio RJ, Wilcox KS (1986) Stimulation of the lateral habenula inhibits dopamine-containing neurons in the substantia nigra and ventral tegmental area of the rat. J Neurosci 6:613-619.

Elliott R, Dolan RJ (1998) Activation of different anterior cingulate foci in association with hypothesis testing and response selection. NeuroImage 8:17-29.

Elliott R, Friston KJ, Dolan RJ (2000) Dissociable neural responses in human reward systems. J Neurosci 20:6159-6165.

Falkenstein M, Hohnsbein J, Hoormann J, Blanke L (1990) Effects of errors in choice reaction tasks on the ERP under focused and divided attention. In: Psychophysiological brain research (Brunia CHM, Gaillard AWK, Kok A, eds), pp 192-195. Tilburg, The Netherlands: Tilburg UP.

Friston KJ, Holmes AP, Worsley KJ, Poline JP, Frith CD, Frackowiak RSJ (1995) Statistical parametric maps in functional imaging: a general linear approach. Hum Brain Mapp 2:189-210.

Friston KJ, Fletcher P, Josephs O, Holmes A, Rugg MD, Turner R (1998) Event-related fMRI: characterizing differential responses. NeuroImage $7: 30-40$.

Garavan H, Ross TJ, Kaufman J, Stein EA (2002) Neuroanatomical dissociation of response conflict from error detection using event-related fMRI. NeuroImage 17:1820-1829.

Gehring WJ, Goss B, Coles MGH, Meyer DE, Donchin E (1993) A neural system for error detection and compensation. Psychol Sci 4:385-390.

Greenhouse S, Geisser S (1959) On methods in the analysis of profile data. Psychometrika 24:95-112.

Holmes AP, Friston KJ (1998) Generalisability, random effects and population inference. NeuroImage 7:754.

Holroyd C, Coles MGH (2002) The neural basis of human error processing: reinforcement learning, dopamine, and the error-related negativity. Psychol Rev 109:679-709.

Horvitz JC (2000) Mesolimbocortical and nigrostriatal dopamine responses to salient non-rewarding events. Neuroscience 96:651-656.

Horvitz JC, Steward T, Jacobs BL (1997) Burst activity of ventral tegmental neurons is elicited by sensory stimuli in the awake cat. Brain Res 759:251-258.

Josephs O, Turner R, Friston K (1997) Event-related fMRI. Hum Brain Mapp 5:243-248.

Lisoprawski A, Herve D, Blanc G, Glowinski J, Tassin JP (1980) Selective activation of the mesocortico-frontal dopaminergic neurones induced by lesion of the habenula in the rat. Brain Res 183:229-234.

Lohmann G, Müller K, Bosch V, Mentzel H, Hessler S, Chen L (2001) LIPSIA-a new software system for the evaluation of functional magnetic resonance images of the human brain. Comput Med Imaging Graph 25:449-457.

Luu P, Tucker DM, Derryberry D, Reed M, Poulsen C (2003) Electrophysiologic responses to errors and feedback in the process of action regulation. Psychol Sci 14:47-53.

Miezin FM, Maccotta L, Ollinger JM, Petersen SE, Buckner RL (2000) Characterizing the hemodynamic response: effects of presentation rate, sampling procedure, and the possibility of ordering brain activity based on relative timing. NeuroImage 11:735-759.

Miltner WHR, Braun CH, Coles MGH (1997) Event-related brain potentials following incorrect feedback in a time-estimation task: evidence for a "generic" neural system for error detection. J Cognit Neurosci 9:788-798. 
Nishikawa T, Fage D, Scatton B (1986) Evidence for and nature of the tonic inhibitory influence of the habenulointerpeduncular pathway upon cerebral dopaminergic transmission in the rat. Brain Res 373:324-336.

Pagnoni G, Zink CF, Montague PR, Berns GS (2002) Activity in the human ventral striatum locked to errors of reward prediction. Nat Neurosci 5:97-98.

Picard N, Strick PL (1996) Motor areas of the medial wall: a review of their location and functional activation. Cereb Cortex 6:342-353.

Rabbitt PMA (1966) Errors and error correction in choice-response tasks. J Exp Psychol 71:264-272.

Reason J (1990) The detection of errors. In: Human error, pp 148-172. Cambridge, UK: Cambridge UP.

Scheibel AB (1997) The thalamus and neuropsychiatric illness. In: The neuropsychiatry of limbic and subcortical disorders (Salloway S, Malloy P, Cummings JL, eds), pp 31-40. Washington, DC: American Psychiatry.

Schultz W (2000) Multiple reward signals in the brain. Nat Rev Neurosci 1:199-207.

Schultz W (2002) Getting formal with dopamine and reward. Neuron 36:241-263.

Schultz W, Dickinson A (2000) Neuronal coding of prediction errors. Annu Rev Neurosci 23:473-500.
Shima K, Tanji J (1998) Role for cingulate motor area cells in voluntary movement selection based on reward. Science 282:1335-1338.

Talairach J, Tournoux P (1988) Co-planar stereotaxis atlas of the human brain. New York: Thieme.

Ullsperger M, von Cramon DY (2001) Subprocesses of performance monitoring: a dissociation of error processing and response competition revealed by event-related fMRI and ERPs. NeuroImage 14:1387-1401.

Vogt BA, Nimchinsky EA, Vogt LJ, Hof PR (1995) Human cingulate cortex: surface features, flat maps, and cytoarchitecture. J Comp Neurol 359:490-506.

Volz KG, Schubotz RI, von Cramon DY (2003) Predicting events of varying probability: uncertainty investigated by fMRI. NeuroImage, in press.

Vorobiev V, Govoni P, Rizzolatti G, Matelli M, Luppino G (1998) Parcellation of human mesial area 6: cytoarchitectonic evidence for three separate areas. Eur J Neurosci 10:2199-2203.

Wansapura JP, Holland SK, Dunn RS, Ball WS (1999) NMR relaxation times in the human brain at 3.0 Tesla. J Magn Reson Imaging 9:531-538.

Worsley KJ, Friston KJ (1995) Analysis of fMRI time-series revisited-again. NeuroImage 2:173-181.

Zarahn E, Aguirre GK, D’Esposito M (1997) Empirical analysis of BOLD fMRI statistics, Vol I: spatially unsmoothed data collected under nullhypothesis conditions. NeuroImage 5:179-197. 